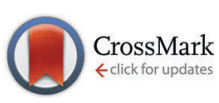

Cite this: Phys. Chem. Chem. Phys., 2015, 17, 9569

DOI: $10.1039 /$ c5cp90039e

www.rsc.org/pccp

\section{Correction: Control of selectivity in allylic alcohol oxidation on gold surfaces: the role of oxygen adatoms and hydroxyl species}

\author{
Gregory M. Mullen, ${ }^{a}$ Liang Zhang, ${ }^{b}$ Edward J. Evans, ${ }^{b}$ Ting Yan, ${ }^{b}$ \\ Graeme Henkelman ${ }^{b}$ and C. Buddie Mullins*abc
}

Correction for 'Control of selectivity in allylic alcohol oxidation on gold surfaces: the role of oxygen adatoms and hydroxyl species' by Gregory M. Mullen et al., Phys. Chem. Chem. Phys., 2015, 17, 4730-4738.

The caption for Fig. 5 in the published article is incorrect. The caption for Fig. 5 should read as follows:

Reaction pathways associated with potential initiation steps for combustion of acrolein on $\mathrm{O} / \mathrm{Au}(111) \mathrm{and} \mathrm{OH} / \mathrm{Au}(111) \mathrm{surfaces}$. Activation barriers and atomic structures were determined via DFT calculations.

The Royal Society of Chemistry apologises for these errors and any consequent inconvenience to authors and readers.

\footnotetext{
${ }^{a}$ McKetta Department of Chemical Engineering, University of Texas at Austin, Austin, Texas 78712-0231, USA. E-mail: mullins@che.utexas.edu

${ }^{b}$ Department of Chemistry, University of Texas at Austin, Austin, Texas 78712-0231, USA

${ }^{c}$ Center for Nano and Molecular Science and Technology, Texas Materials Institute, and Center for Electrochemistry, University of Texas at Austin, Austin, Texas 78712-0231, USA
} 